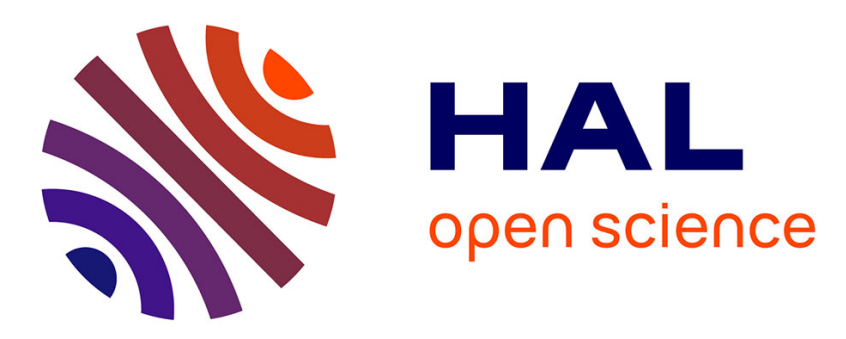

\title{
Numerical simulation of NACA4412 airfoil in pre-stall conditions
}

\author{
Vincent Gleize, Michel Costes, Ivan Mary
}

\section{To cite this version:}

Vincent Gleize, Michel Costes, Ivan Mary. Numerical simulation of NACA4412 airfoil in pre-stall conditions. 55th 3AF International Conference on Applied Aerodynamics, Mar 2021, Poitiers, France. hal-03224335

\section{HAL Id: hal-03224335 \\ https://hal.science/hal-03224335}

Submitted on 11 May 2021

HAL is a multi-disciplinary open access archive for the deposit and dissemination of scientific research documents, whether they are published or not. The documents may come from teaching and research institutions in France or abroad, or from public or private research centers.
L'archive ouverte pluridisciplinaire HAL, est destinée au dépôt et à la diffusion de documents scientifiques de niveau recherche, publiés ou non, émanant des établissements d'enseignement et de recherche français ou étrangers, des laboratoires publics ou privés. 


\title{
NUMERICAL SIMULATION OF NACA4412 AIRFOIL IN PRE-STALL CONDITIONS
}

\author{
V. Gleize ${ }^{(1)}$, M. Costes ${ }^{(2)}$ and I. Mary ${ }^{(3)}$ \\ (1) ONERA, The French Aerospace Lab, F-92190 Meudon, France, vincent.gleize@onera.fr \\ (2) ONERA, The French Aerospace Lab, F-92190 Meudon, France, michel.costes@onera.fr \\ (3) ONERA, The French Aerospace Lab, F-92320 Châtillon, France, ivan.mary@onera.fr
}

\begin{abstract}
DNS simulations of the NACA 4412 airfoil in pre-stall conditions have been completed. The Reynolds number based on airfoil chord and freestream velocity is equal to 0.35 Millions, and the freestream Mach number to 0.117 . Transition is triggered on both upper and lower surface for avoiding the occurrence of laminar separation bubbles and to ensure turbulent mixing in the wake. Three incidences have been considered, 5, 8 and 10 degrees. The results obtained show a fairly good correlation of the present simulations with classical MSES airfoil simulations, both in terms of pressure and skin-friction distribution, with an earlier and more extended flow separation in the DNS. The database thus generated will be deeply analysed and enriched for larger incidences in the future.
\end{abstract}

\section{INTRODUCTION}

Stall is a very important safety issue for civil aviation. During the design / development phase of an aircraft, the safety margins imposed by this severe flight condition restrict the flight envelope of the aircraft, directly or indirectly by its consequences on the sizing of the aircraft, e.g. the high-lift systems required during take-off and landing. Moreover, in order to better control the aircraft behaviour in flight and avoid unexpected phenomena, flow separation may be deliberately triggered by "stall strips", "vortillons" or similar devices, leading to an increase in drag and therefore of fuel consumption in cruise. From the operational point of view, upset and loss of control in flight events reported during the last years put the emphasis on the need to train pilots to react well in these flight situations in order to recover rapidly and avoid fatalities. This makes it necessary to develop flight simulators capable of training pilots in these highly nonlinear working conditions, and thus requires the capability to describe the behaviour of the aircraft under such non-conventional flight conditions. In addition, upset recovery control algorithms, which are currently in the research domain, also need this kind of description of the behaviour of the airplane. An important aspect of the problem concerns pre-stall conditions, when flow separation is initiated at the trailing-edge of the wing, leading to potential loss of control surface efficiency. Most of these points require predicting the aerodynamic flow field accurately, more especially as far as the development of flow separation is concerned. From the experimental point of view, accurate wind-tunnel testing of aircraft near stall is a difficult task for several reasons. The high attitude angles encountered in such flight conditions lead to large wind-tunnel wall effects, high blockage, so that only small scale models can be tested. This leaves a large uncertainty on the effect of Reynolds number on measured data. Furthermore, aircraft flying in the stall regime encounter time-varying flow conditions with large variations of the working conditions so that dynamic testing is required to be representative. Finally, stalled flows themselves have a large degree of unsteadiness, so that wind-tunnel models are subject to large static and dynamic loads, rendering measurements even more difficult. As a result, it is expected to call more and more for numerical predictions for simulating aircraft at stall. In principle, a direct simulation of the Navier-Stokes equations (DNS) can handle the problem. However, as aeronautical flows occur at very large Reynolds numbers (of the order of $10^{7}-10^{8}$ ), this methodology is not applicable. Large Eddy Simulation (LES) methods, although less general than DNS as they account for the large scales of turbulence 
only, require mesh refinements still prohibitive in the near-wall region for these Reynolds numbers. Finally, the progress of hybrid RANS / LES and Wall-Modelled LES methods now makes it possible to approach realistic Reynolds numbers, but, even when considered as mature, they are not fully operational on the stall problem yet. Therefore only the Reynolds averaged Navier-Stokes equations (RANS) are currently applicable on realistic configurations. Unfortunately, because of the limitations associated with existing turbulence models, RANS simulations are not able to predict the occurrence of flow separation with sufficient precision.

These motivations prompted ONERA to define the PANDA federating research project (PRF) in order to address various aspects of the stall problem. Among these, the objective of the present work is to perform advanced DNS-type simulations on a wing section for the entire polar range from the fully attached regime to the deep stall one in order to build a reference database for improved RANS and hybrid RANS / LES modelling. As several stall mechanisms are susceptible to occur, it was chosen to simplify the problem by favoring turbulent flow separation which starts from the trailing edge of lifting surfaces. As a result, the NACA 4412 airfoil was selected as test case. Such an airfoil effectively shows trailing edge separation, as can be found on the NASA Turbulence Modeling Resource https://turbmodels.larc.nasa.gov/naca4412sep_val.html web site. Furthermore, this airfoil has also been considered by Schlatter et al. in a series of publications, essentially concentrating on a single incidence ( $5 \mathrm{deg}$.) and varying the Reynolds number in the range $\left[10^{5}, 10^{6}\right]$ to investigate the effect of adverse pressure gradient on the boundary layer $[5,11,12,14,13,10]$. In our case, the Reynolds number based on airfoil chord is equal to $3.510^{5}$, the free-stream Mach number to 0.117 , and three incidences in the pre-stall regime are considered.

\section{METHODOLOGY}

\subsection{HPC Numerical method}

The ONERA FastS solver has been used to solve the compressible unsteady Navier-Stokes equations, in a finite volume approach over structured grids. No subgridscale model is used so that the method can be viewed either as an implicit LES simulation (ILES) or as a DNS one according to the mesh resolution used. The spatial scheme for the convective term is the one proposed in [7]. This scheme is second-order accurate and it allows to nullify locally the numerical dissipation whenever a numerical wiggle is not detected. The time integration is carried out by means of the second-order accurate backward scheme of Gear. An approximate Newton method is employed to solve the non-linear problem. The usual
Jameson-Yoon approximation of the Jacobian matrix is retained, whereas the LU-SGS algorithm allows to solve the linear system. Moreover a Newton algorithm with a spatially adaptive sub-iteration number is used to optimise the computational time $[2,6]$.

The FastS solver relies on an hybrid MPI/OpenMP framework, where the memory is distributed (by distributing CFD grids) on the processors at high level, i.e., between nodes, whereas multithreading is managed via OpenMP within a given node. HPC optimization has been performed within an Intel collaboration to allow a better use of cache memories and vector units of KNL processors: it enables to update approximately 4 million cells per second per core.

\subsection{Description of mesh}

Such kind of simulation requires capturing all turbulent scales from the most energetic large eddies to the smaller ones where viscous dissipation is effective. The number of mesh points required for doing so increases exponentially with Reynolds number and accounting for the number of time steps necessary to converge the solution statistically, an order of magnitude more CPU resources is necessary. Furthermore, the spanwise extension of the mesh is getting larger with the thickening of the boundary layer. At stall, a span of more than one wing chord is necessary, leading to very large numbers of mesh cells. Therefore, only moderate Reynolds numbers are still accessible for DNS simulations. In our case, the Reynolds number that is being investigated is equal to $0.3510^{6}$. Such a value is significantly lower than those actually encountered in flight, but the goal is to generate reference data for the analysis and improvement of turbulence models.

The structured mesh was generated with Pointwise. For efficiency, the flow domain is split into a turbulent part, overlapping the boundary layer and the wake, where a true 3D DNS mesh is considered, and a "potential" part outside where the mesh is actually $2.5 \mathrm{D}$ only (figure 1 ). Partial $1 / N, N>1$ matching of the 2.5D and 3D meshes is thus ensured in a conservative way along the spanwise direction, with $N=20$ in the present case, thus allowing a large saving of mesh points. The DNS part of the mesh follows the recognised criteria for such simulations: $\Delta x^{+} \leq 12, \Delta y^{+} \leq 1, \Delta z^{+} \leq 6$. The distribution of these quantities based on the first mesh cell on the upper surface is plotted in figure 2 for the 5 degree angle of attack case. As a cell-centered approach is used, the plotted $y^{+}$ values at the wall should be divided by two to be representative of the wall discretisation. Additionally, the mesh size along the longitudinal direction is kept constant and equal to that on the airfoil over one chord downstream the trailing edge. The wall-normal stretching ratio is also maintained below 1.02. The spanwise extension of the mesh depends on the angle of attack considered, as it 
should not constrain the development of flow separation. This was obtained by building an original mesh of span equal to $0.78 \%$ chord, and the final mesh is obtained by stacking the required number of elementary meshes along the spanwise direction for obtaining the desired span. For doing so, elementary mesh translation and corresponding blocks connection was completed with the Cassiopée preand post-processing tool developped by ONERA [1]. As a result, the mesh extended over $30 \%$ chord at 5 deg. incidence, and over $40 \%$ chord at 8 and $10 \mathrm{deg}$. In these last two cases, the complete mesh thus included $1.6810^{9}$ mesh cells, making good HPC capabilities mandatory to run the corresponding cases. A comparison of the vertical mesh spacing with an estimated lower bound of the Kolmogorov scale is plotted in figure 3 for the upper surface at $5 \mathrm{deg}$. incidence. This Kolmogorov scale is computed in the log region of the boundary layer as $\left(\kappa y^{+}\right)^{1 / 4}$ and provides a reliable lower bound of this quantity in the whole boundary layer at zero pressure gradient (see [3] and [9], pp. 67-68). It can thus be expected that the present grid fineness is capable to capture the full range of turbulent fluctuations for the Reynolds number considered.

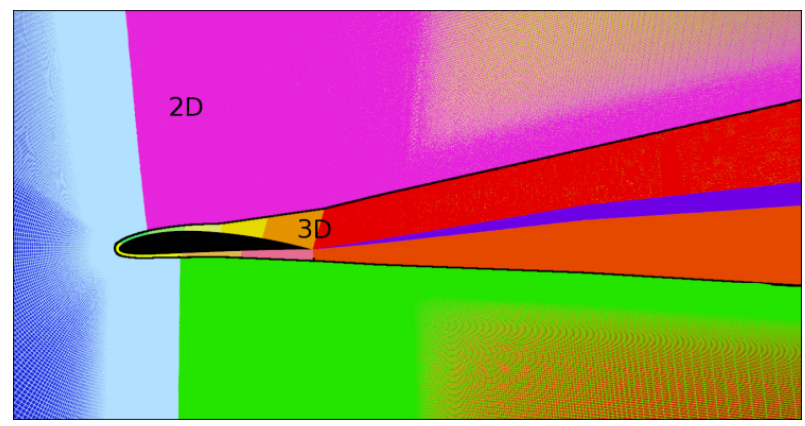

Figure 1: Overview of mesh

\subsection{Transition triggering}

As the present work aims to consider turbulent flow separation from the trailing edge, it was necessary to trigger laminar-turbulent transition on both upper and lower surfaces of the airfoil. On the upper surface, the goal is to avoid the occurrence of laminar separation bubbles near the leading edge, so that the transition should be ensured as close as possible from the leading edge. On the other hand, the transition on the lower surface should be triggered before the trailing edge in order to get the mixing of two turbulent boundary layers in the near wake. For both upper and lower surface, this is not an easy task as the Reynolds number is in the critical range where a large amount of natural laminarity develops over the airfoil, roughly half a chord on the upper surface and the whole lower surface. Furthermore, it is also highly recommended that the transition triggering mechanism

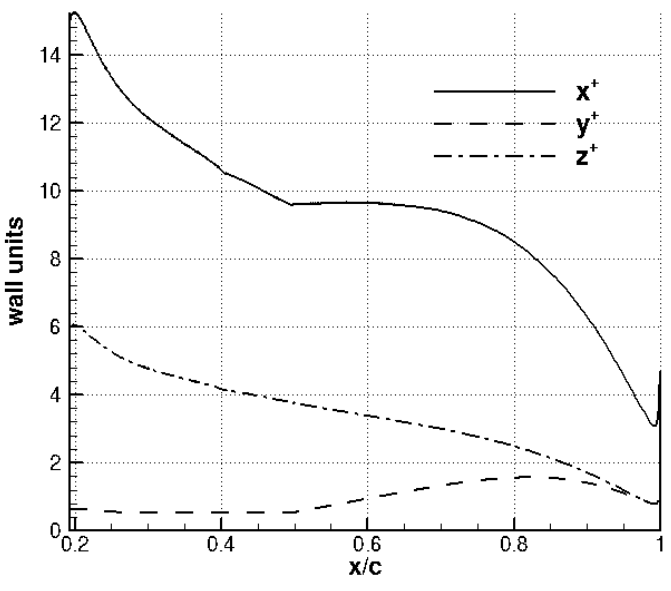

Figure 2: Distribution of mesh cell sizes on the upper surface of the airfoil

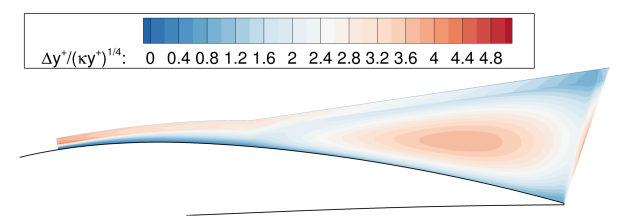

Figure 3: Upper surface mesh spacing estimated in terms of lower bound of Kolmogorov scale

should disturb as little as possible the flow field. This is why the procedure proposed by Deck et al. [4] was adopted as it was found to generate very little acoustic flow perturbations while allowing a fast transition to turbulence. It consists in small cylindric roughness elements equidistantly distributed in the spanwise direction (see figure 4). Their height and spanwise distance were determined in order to maximize the transient growth of the corresponding perturbations, typically leading to a height of $60 \%$ and a spacing of $100 \%$ in terms of local boundary layer thickness. These cylinders are represented in the DNS mesh by a simple IBC approach, similarly to what is described in [4].

The determination of the chordwise position where to set the cylinders was done by trial and error from numerical simulations over one single elementary mesh described earlier. On the upper surface, the transition was finally set at $15 \%$ chord at $5 \mathrm{deg}$. incidence, and at $5 \%$ chord at 8 and $10 \mathrm{deg}$. For all cases, lower surface transition was set at $50 \%$ chord.

A view of the transition process is plotted in figure 5 at $5 \mathrm{deg}$. angle of attack. It shows an iso-surface of the 
Q-criterion coloured by density. A horseshoe vortex is created around each cylinder. Right downstream the array of cylinders, elongated vortex structures are thus obtained, separating the areas of flow acceleration between the cylinders and low velocity regions due to the recirculation downstream the cylinders. These longitudinal structures in the vicinity of the wall interact and connect downstream where hairpin vortex structures can be noted further from the wall. Instabilities then rapidly develop with the occurrence of 3D turbulent spots which contaminate the whole boundary layer and transition to turbulence is settled. Such a mechanism is also at hand on the lower surface, and works similarly at higher incidences too.

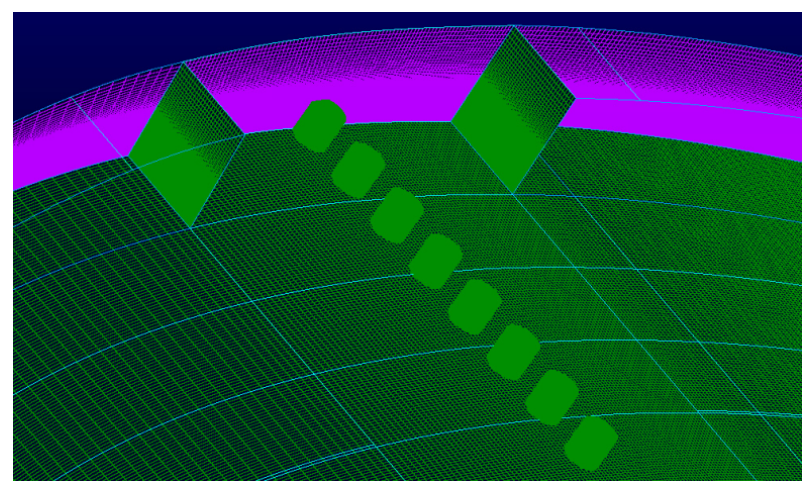

Figure 4: View of cylinders triggering transition

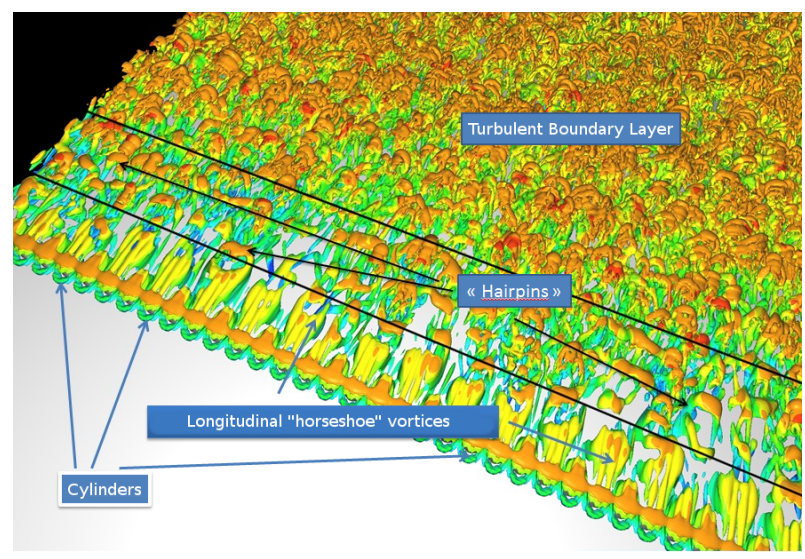

Figure 5: View of transition development on upper surface at 5 deg. incidence

\section{RESULTS}

\subsection{Introduction}

All three incidences were initialised from a RANS solution at the same angle of attack. The time step used was identical in all cases, equal to $\Delta t U_{\infty} / c=6.210^{-5}$ convective time units. A maximum of 30 Newton iterations are used at each time step to converge the nonlinear implicit system. The computations were run for five convective times $c / U_{\infty}$ in order to set the turbulent mean flow and remove transient effects. Once the solution is stabilized around a constant mean state, the flow is statistically averaged over twenty additional convective times and samples recorded versus time at selected locations of the flow-field for post-processing.

Snapshots of the solutions during this phase of the simulation are presented in figures 6,7 and 8 for 5 deg., 8 deg. and $10 \mathrm{deg}$. incidence respectively, showing an isosurface of the Q-criterion. At 5 deg. incidence, the Qcriterion is coloured by density. The later triggering of transition on the upper surface with respect to the two other cases can be noted. Furthermore, the flow remains attached whatever the time of the simulation considered. At 8 and $10 \mathrm{deg}$. incidence, trailing edge separation begins to develop, which is highlighted by the blue region near the trailing edge where reversed flow is obtained as the $\mathrm{Q}$ iso-surface is now coloured by axial velocity. The increased flow separation with angle of attack is clearly noticeable too. For all cases, upper and lower surface transition appear to be correctly triggered with a rich turbulent content of the subsequent boundary layer.

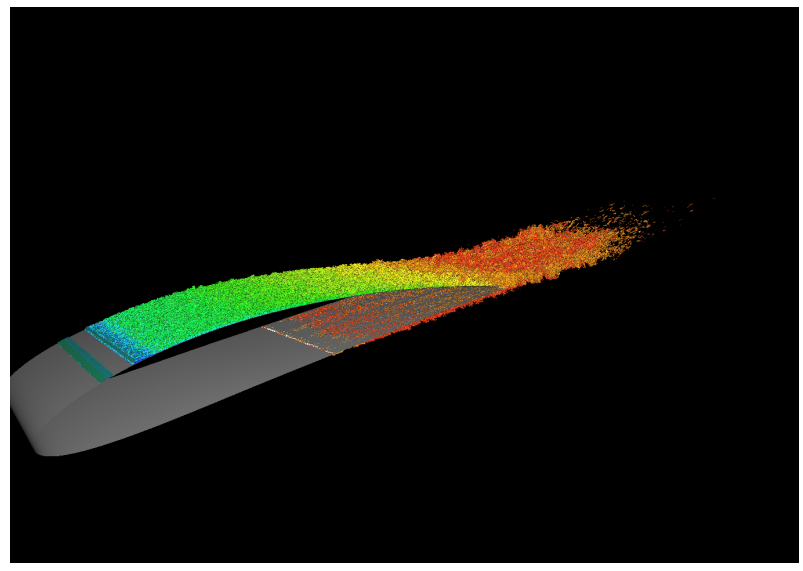

Figure 6: Contours of Q criterion coloured by density at $5 \mathrm{deg}$. incidence.

\subsection{Comparison of mean flow characteris- tics}

The mean contours of Mach number and flow streamlines are plotted in figures 9, 10 and 11 for the three incidences considered. The occurrence of trailing edge separation at $8 \mathrm{deg}$. incidence and the growth of the recirculation when increasing the angle of attack to $10 \mathrm{deg}$. can be noted, due to a growing adverse pressure gradient as larger leading edge suction is obtained with increasing incidence. On the contrary, the flow remains attached all along the air- 


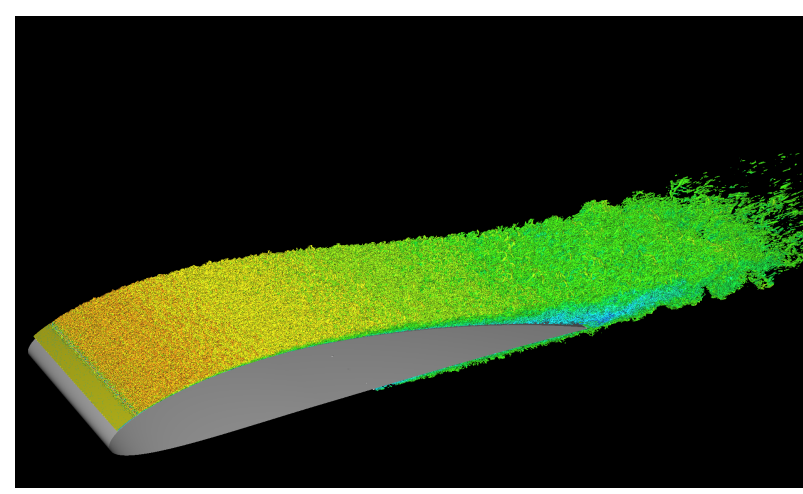

Figure 7: Contours of Q criterion coloured by longitudinal velocity at $8 \mathrm{deg}$.incidence.

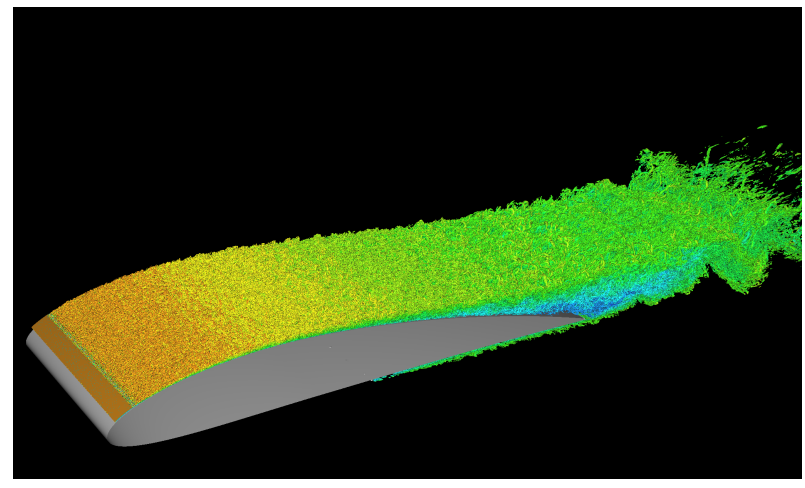

Figure 8: Contours of Q criterion coloured by longitudinal velocity at $10 \mathrm{deg}$. incidence.

foil upper surface at $5 \mathrm{deg}$. incidence and, for all cases, on the lower surface too.

As a result, turbulent fluctuations in the rear part of the airfoil boundary layer increase with angle of attack as shown by the contours of turbulent kinetic energy normalized by freestream velocity in figures 12, 13 and 14 . A first maximum of this quantity can be seen in the transition region, with little dependence to the airfoil incidence as it is mainly related to the transition triggering mechanism. On the contrary, two other maxima of intensity growing with incidence are found in the very near wake close to the trailing edge. The first one comes from the upper surface boundary layer. This maximum is moving upwards as the boundary layer is thickening due to the larger pressure gradient and flow separation. Consistently, the turbulent kinetic energy also increases significantly more upstream in the boundary layer over the airfoil. Maximum values are located in the shear layer between the low-speed region near the airfoil wall and the external flow. Therefore the turbulent kinetic energy remains small in the recirculation bubble when separation occurs at 8 and $10 \mathrm{deg}$. incidence, extending upwards the low-turbulence zone found near the trailing edge in the near-wall region of the attached boundary layer at $5 \mathrm{deg}$. incidence. The second maximum in the wake is coming from the lower surface of the airfoil. Although the lower surface boundary layer characteristics are not expected to vary much between the three cases as the negative chordwise pressure gradient remains small, this maximum also strongly increases with angle of attack. It is probably due to the interaction between the two boundary layers as they mix in the wake.

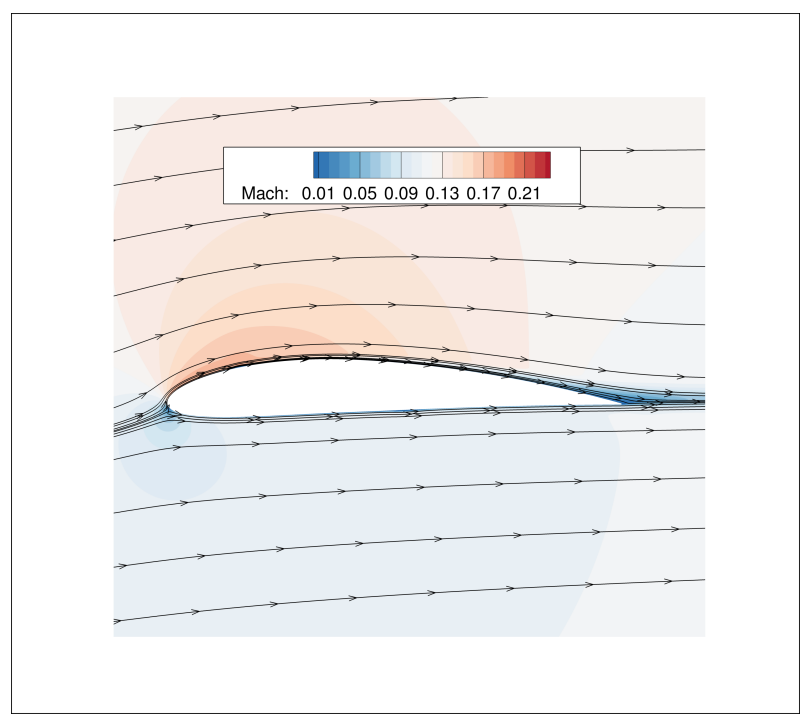

Figure 9: Mach number contours and streamlines of the time-averaged flow at $5 \mathrm{deg}$. incidence.

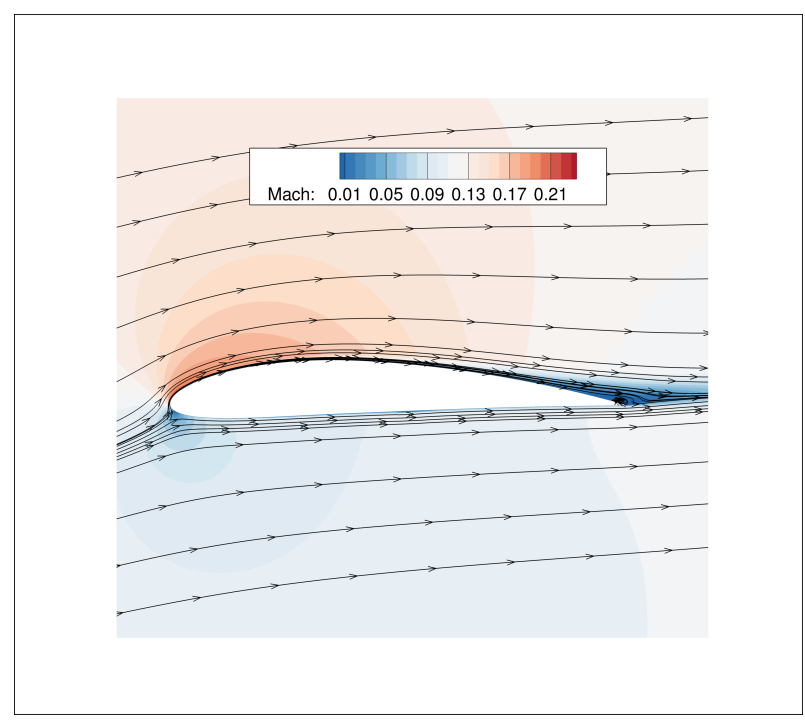

Figure 10: Mach number contours and streamlines of the time-averaged flow at $8 \mathrm{deg}$. incidence. 


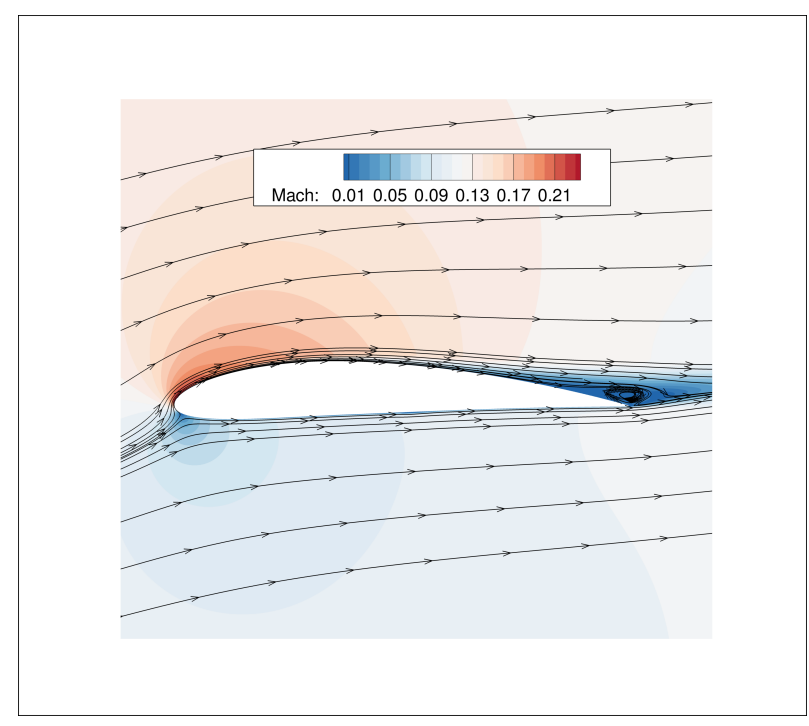

Figure 11: Mach number contours and streamlines of the time-averaged flow at $10 \mathrm{deg}$. incidence.

\subsection{Reynolds stresses}

The Reynolds stresses obtained from statistical averaging are presented in figures 15, 16 and 17 for 5 deg., 8 deg. and $10 \mathrm{deg}$. incidence respectively. They are all normalized by the local value of kinetic energy to compare with the well-known equilibrium values of these quantities (e.g. see Pope [8], pp. 281-285). The three normal stresses as well as the shear stress are presented. As far as the shear-stress is concerned, only its modulus has been considered in the plots as they have an opposite sign on the upper and lower surface (as expected they are negative on the upper surface). For all cases, the first normal stress component $u^{\prime 2} / k$ shows a value slightly above 1 downstream transition triggering. This value then progressively reduces to get below 0.9 when progressing from upstream towards the trailing-edge, and this happens all the more early that the angle of attack increases: it occurs around $56 \%$ chord at $5 \mathrm{deg}$., $51 \%$ at $8 \mathrm{deg}$. and $50 \%$ at $10 \mathrm{deg}$. As far as the other normal stresses are concerned along this same path, the second component $v^{\prime 2} / k$ increases from 0.3 to 0.5 , and the third one $w^{2} / k$ keeps a fairly constant value slightly above 0.6 . Although hardly visible on the figures, close to the wall $u^{\prime 2} / k$ remains also quite high with values above 1.4, except on the upper surface in the adverse pressure gradient region when approaching the trailing edge. There, the near-wall values drop between 1.3 and 1 over a zone extending more and more upstream as the angle of attack is increased, indicating a decreased production of turbulence there. However, in the flow recirculation, near-wall values increase again above 1.1. As far as the values of $v^{\prime 2} / k$ and $w^{2} / k$ are concerned near the wall, they are below 0.1 and 0.4 respectively in the upstream part of the boundary layer, and

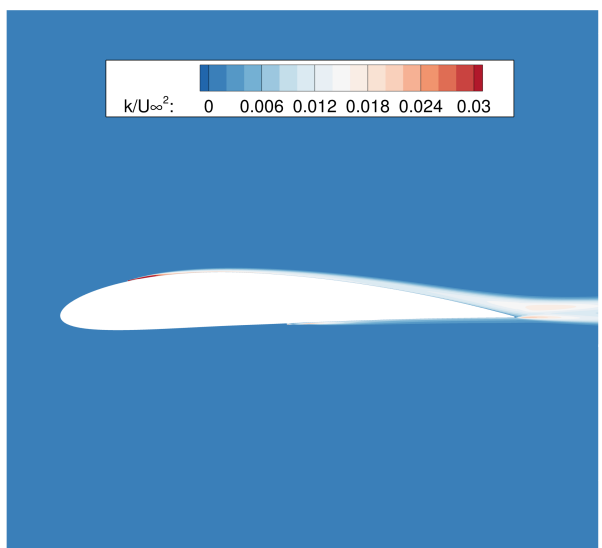

Figure 12: Contours of turbulent kinetic energy normalized by freestream velocity for the time-averaged flow at $5 \mathrm{deg}$. incidence.

they increase progressively when approaching the trailing edge. The shear-stress $u^{\prime} v^{\prime} / k$ shows a fairly constant value in a large part of the boundary layer with a magnitude slightly above 0.3 , except in the transition region where it is much lower. Near wall values are also lower, of the order of 0.1 . Globally the order of magnitude of the Reynolds stresses lie in the expected range, both nearwall and further from the wall. The larger the pressure gradient, the further the Reynolds stresses are from classical values. As a matter of fact, the larger deviations occur in the near wake where the magnitude of the three normal stresses get much closer to one another.

\subsection{Comparison of mean results with MSES simulation}

The time averaged surface data was compared with numerical results obtained with the classical MSES software (https://tlo.mit.edu/technologies/mses-softwarehigh-lift-multielement-airfoil-configurations), which solves the Euler equations coupled with boundary layer and is a classical tool used in airfoil design. The chordwise pressure and skin-friction distributions are presented in figures 18, 19 and 20 for the pressure, and in figures 21, 22 and 23 for the skin-friction.

Globally, the pressure distributions computed by both methods match reasonably well for the three incidences considered (figures 18, 19, 20). Yet the effect of the transition triggering device is clearly noticeable on the pressure distribution computed with FastS: large local pressure perturbations are found in the vicinity of the location of the roughness triggering transition, for both upper and lower surface. Nevertheless, the influence of 


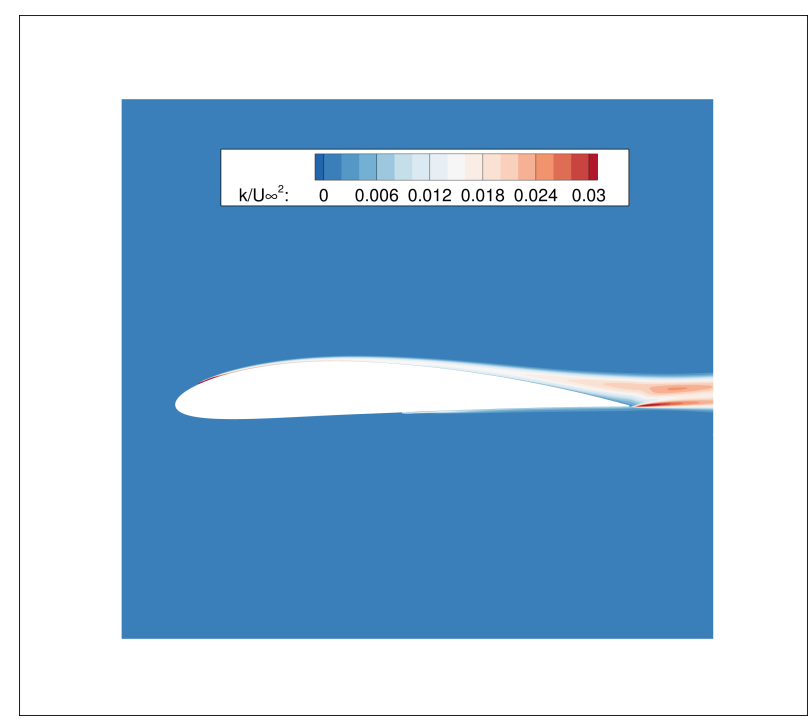

Figure 13: Contours of turbulent kinetic energy normalized by freestream velocity for the time-averaged flow at 8 deg. incidence.

the corresponding array of cylinders remains local as the pressure distributions show gradients very similar to those obtained with MSES outside the interval $\pm 10 \% c$ around the transition triggering position. A pressure perturbation, yet of much smaller amplitude, can also be noted at the prescribed transition in MSES.

However, the main difference between FastS and MSES results is that FastS consistently predicts lower differential pressure between the upper and lower surface of the airfoil than MSES for all considered incidences. The difference in pressure prediction increases with the angle of attack. This is clearly noticeable for the upper surface, more especially the leading-edge suction, but also on the lower surface of the airfoil. The trailing edge pressure predicted by MSES is also always higher than that computed with FastS, whether the flow is locally attached (5 deg.) or separated (8 and $10 \mathrm{deg}$.). Furthermore, for a given angle of attack, the flow separation extends over a larger region in FastS predictions with a pressure plateau better defined than in MSES results.

The larger flow separation predicted by FastS is confirmed by the comparison of skin-friction distribution (figures 21, 22, 23), as the skin-friction on the upper surface goes to zero earlier in FastS predictions at 8 deg. (94\% $c$ with FastS, $100 \% c$ with MSES) and 10 deg. (86\% $c$ with FastS, 95\% $c$ with MSES). Again, the global agreement between the predicted distributions of skin-friction is reasonably good. The values of skin-friction are almost identical in the laminar regions of the flow, and their matching downstream transition is also correct.

The biggest difference again comes from the transition region, because of the effect of the array of cylinders

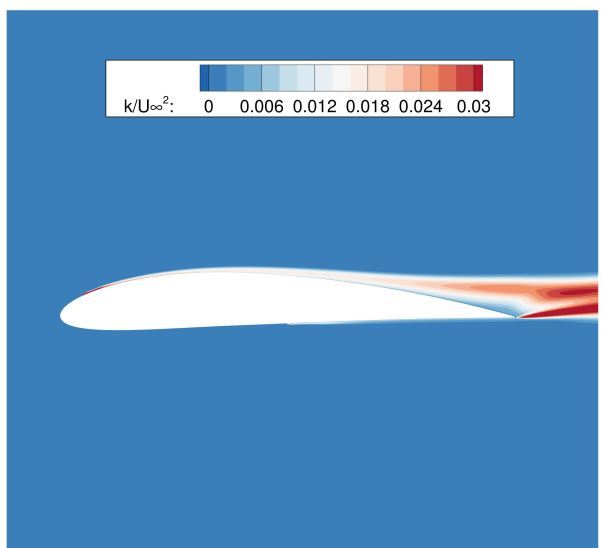

Figure 14: Contours of turbulent kinetic energy normalized by freestream velocity for the time-averaged flow at $10 \mathrm{deg}$. incidence.

used to trigger transition in FastS simulations. As described above, the cylinders induce local flow separation downstream, and the spanwise averaging of airfoil surface data leads to a complex pattern, which, on the upper surface at $5 \mathrm{deg}$. incidence, may be interpreted as a succession of two small recirculation bubbles before a turbulent boundary layer begins to develop. The first one is materialised by the large peak of skin-friction modulus at $15 \%$ c. A corresponding peak can also be noted on the lower surface at mid-chord as a trace of similar phenomena. The transition happens faster at 8 and $10 \mathrm{deg}$. incidence because a larger adverse pressure gradient occurs, but at least one recirculation can be distinguished on the time- and span-averaged surface data, its signature corresponding again to the peak in $C_{f}$ at the location of the cylinders, i.e. 5\% $c$ on the upper surface and $50 \% c$ on the lower surface. This transition triggering device also affects the skin-friction over roughly $10 \% c$ downstream on the upper surface, with a $C_{f}$ value significantly larger in the FastS predictions than in MSES ones. However, as already mentioned, computed values further downstream are in fairly good agreement so that the turbulent boundary layer seems not to be much affected by the way transition was triggered. The effect of the transition triggering device seems to extend downstream over a larger extent on the lower surface (about 15\% c), probably because turbulence is much more difficult to trigger due to the very low pressure gradient in this area of the airfoil. However, as the objective of triggering transition on the lower surface of the airfoil is to get turbulent mixing of boundary layers at the trailing edge, such a difference is not very important. 


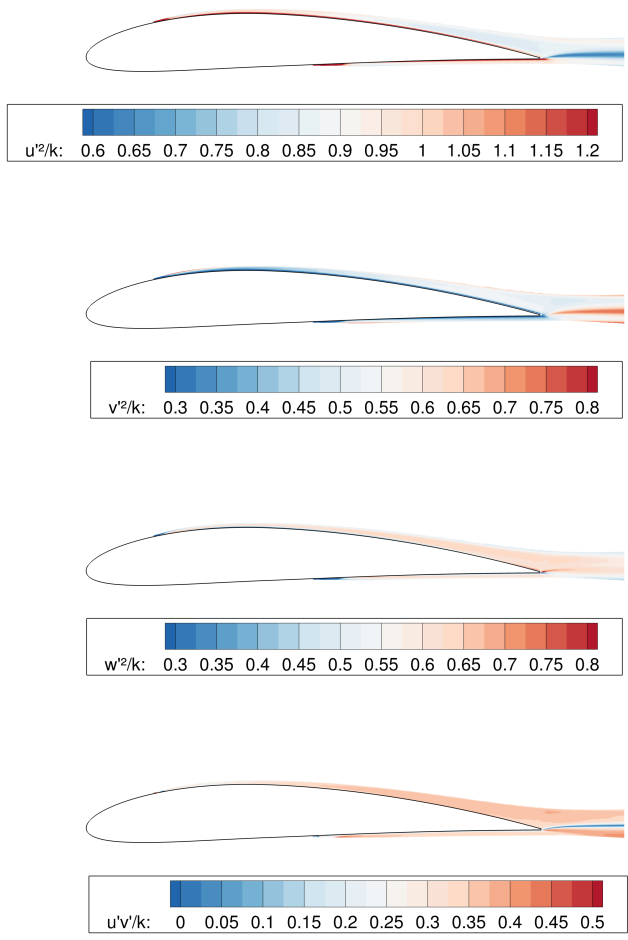

Figure 15: Contours of Reynolds stresses at $5 \mathrm{deg}$.

The lift and drag polars versus angle of attack computed by MSES are compared to the results obained with FastS at the three same incidences in figures 24 and 25 respectively. FastS results also show an error bar based on the standard deviation computed for the whole recorded signal around the corresponding mean value. Consistently with the comparison of pressure and skin-friction, FastS predicts lower lift than MSES at all angles of attack considered. The difference is small at $5 \mathrm{deg}$. incidence, but it is getting larger as the angle of attack is increased, so that the lift curve slope is lower in FastS predictions than in MSES ones. In any case, this difference is much larger than standard deviation of the unsteady lift computed with FastS. As far as the drag polar is concerned, FastS also predicts a larger drag rise after $5 \mathrm{deg}$. incidence than MSES. Again, this is consistent with the larger extent of flow separation obtained with FastS.

\section{CONCLUSIONS}

The direct numerical simulation of the flow around a NACA 4412 airfoil was presented for three incidences in the pre-stall regime. The Reynolds number based on freestream velocity and airfoil chord is equal to $0.35 \mathrm{Mil}-$ lion, and the freestream Mach number to 0.117. The objective is to study turbulent flow separation at the airfoil

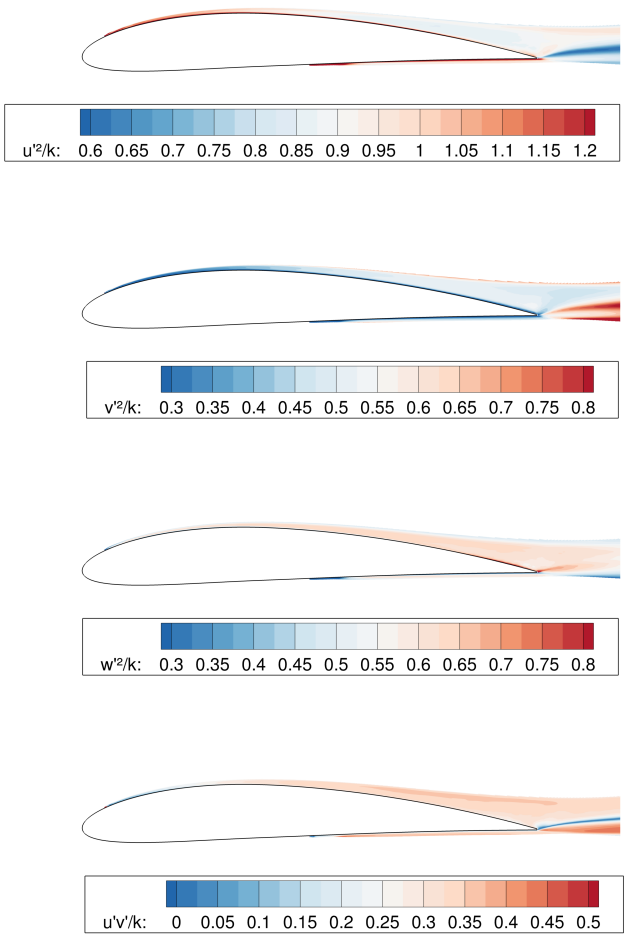

Figure 16: Contours of Reynolds stresses at $8 \mathrm{deg}$.

trailing edge. Great attention was put in mesh generation for capturing the turbulent scales of the flow. Another difficulty is to implement laminar-turbulent transition triggering properly, in order to avoid the occurrence of laminar separation bubbles at the leading edge and to ensure turbulent mixing of the upper surface and lower surface boundary layers in the wake. The results obtained so far show that these two objectives were met. The incidences computed up to now are the following: 5,8 and 10 degrees. All cases were started from a RANS solution as initial condition. Five convective time units were judged necessary for damping transient effects. Flow data was subsequently recorded over twenty additional convective time units for obtaining mean flow statistics. The results obtained compare reasonably well with MSES predictions in terms of airfoil surface data, although DNS shows an earlier and more extended occurrence of trailing edge flow separation. The distribution of RMS data and Reynolds stresses in the boundary layer also yields values in the expected range.

Future work will focus on a detailed analysis of the large amount of data produced by these simulations in order to determine the characteristics of the turbulent boundary layer for large pressure gradients when flow separation occurs. The present simulations will also be pursued for larger angles of attack with more severe flow separation. Finally, RANS simulations will also be completed 

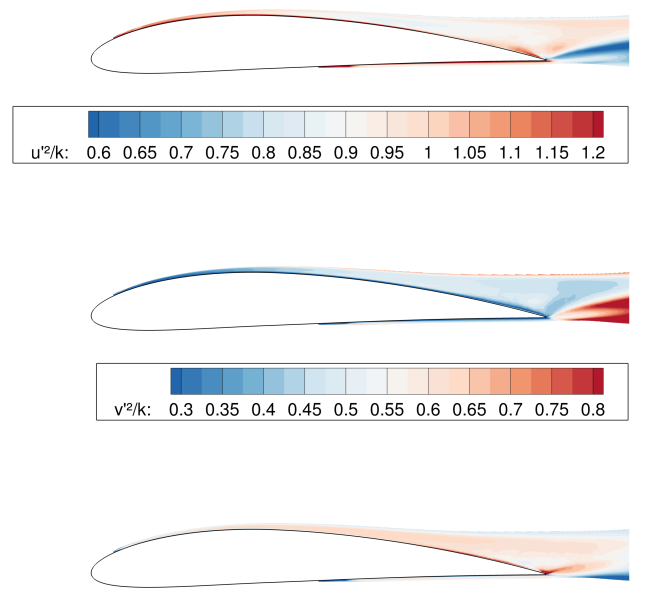

\begin{tabular}{llllllllllllllll}
\hline $\mathrm{w}^{\prime 2} / \mathrm{k}:$ & 0.3 & 0.35 & 0.4 & 0.45 & 0.5 & 0.55 & 0.6 & 0.65 & 0.7 & 0.75 & 0.8 \\
\hline
\end{tabular}

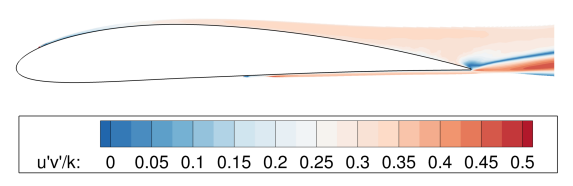

Figure 17: Contours of Reynolds stresses at $10 \mathrm{deg}$.

for comparison and improvement of turbulence models for this difficult type of flows.

\section{ACKNOWLEDGEMENTS}

The numerical simulations presented in this work were partly performed with the support of GENCI (Grand Etablissement National de Calcul Intensif) on Irene KNL supercomputer.

\section{REFERENCES}

[1] C. Benoit, S. Péron, and S. Landier. Cassiopee: A cfd preand post-processing tool. Aerospace Science and Technology, 45:272-283, 2015.

[2] F. Daude, I. Mary, and P. Comte. Self-adaptive newtonbased iteration strategy for the les of turbulent multi-scale flows. Computers \& Fluids, 100:278-290, 2014.

[3] C. M. de Silva, E. P. Gnanamanickam, C. Atkinson, N. A. Buchmann, N. Hutchins, J. Soria, and I. Marusic. High spatial range velocity measurements in a high reynolds number turbulent boundary layer. Physics of fluids, 26(2):025117, 2014.

[4] S. Deck, P.-E. Weiss, and N. Renard. A rapid and low noise switch from RANS to WMLES on curvilinear grids with compressible flow solvers. Journal of Computational Physics, 363:231-255, 2018.

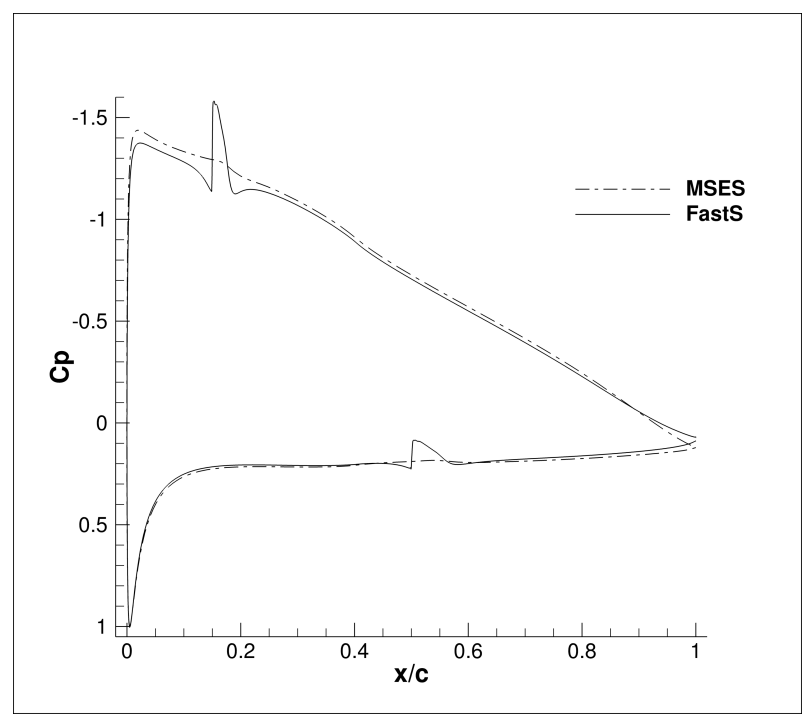

Figure 18: Comparison of mean Cp computed with FastS and MSES at $5 \mathrm{deg}$. incidence.

[5] S. M. Hosseini, R. Vinuesa, A. Schlatter, P. Hanifi, and D. S. Henningson. Direct numerical simulation of the flow around a wing section at moderate reynolds number. International Journal of Heat and Fluid Flow, 61:117-128, 2016.

[6] G. Jeanmasson, I. I. Mary, and L. Mieussens. On some explicit local time stepping finite volume schemes for cfd. Journal of Computational Physics, 397:108818, 2019.

[7] I. Mary and P. Sagaut. Large eddy simulation of flow around an airfoil near stall. AIAA journal, 40(6):1139$1145,2002$.

[8] S. B. Pope. Turbulent Flows. Cambridge University Press, 2000.

[9] Nicolas Renard. Simulations numériques avancées et analyses physiques de couches limites turbulentes à grand nombre de Reynolds. PhD thesis, 2016.

[10] Á. Tanarro, R. Vinuesa, and P. Schlatter. Effect of adverse pressure gradients on turbulent wing boundary layers. Journal of Fluid Mechanics, 883, 2020.

[11] R. Vinuesa, A. Bobke, R. Örlü, and P. Schlatter. On determining characteristic length scales in pressuregradient turbulent boundary layers. Physics of fluids, 28(5):055101, 2016.

[12] R. Vinuesa, S. M. Hosseini, A. Hanifi, D. S. Henningson, and P. Schlatter. Pressure-gradient turbulent boundary layers developing around a wing section. Flow, turbulence and combustion, 99(3-4):613-641, 2017.

[13] R. Vinuesa, P. S. Negi, M. Atzori, A. Hanifi, D. S. Henningson, and P. Schlatter. Turbulent boundary layers around wing sections up to rec $=1,000,000$. International Journal of Heat and Fluid Flow, 72:86-99, 2018.

[14] R. Vinuesa, R. Örlü, and P. Schlatter. Characterisation of backflow events over a wing section. Journal of Turbulence, 18(2):170-185, 2017. 


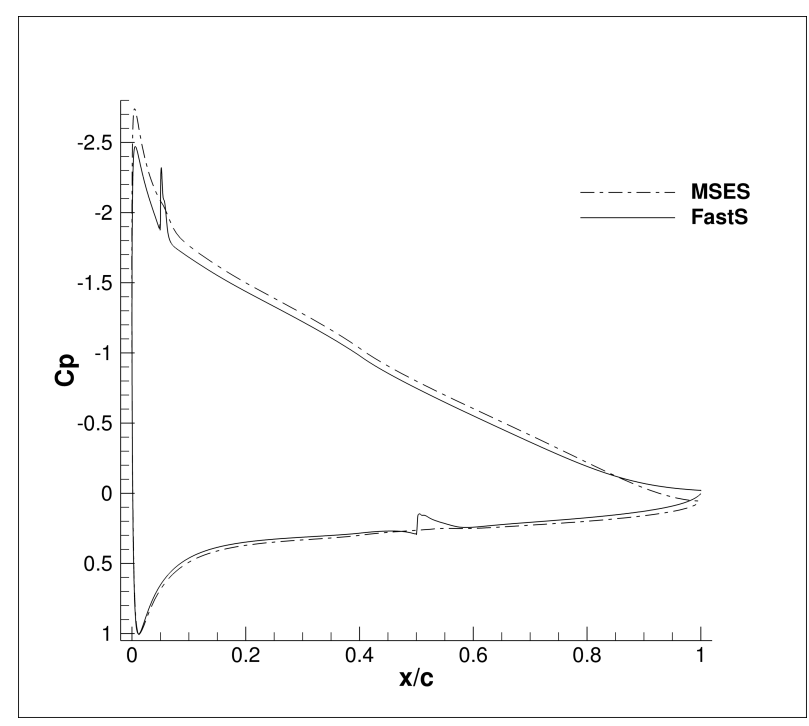

Figure 19: Comparison of mean Cp computed with FastS and MSES at $8 \mathrm{deg}$. incidence.

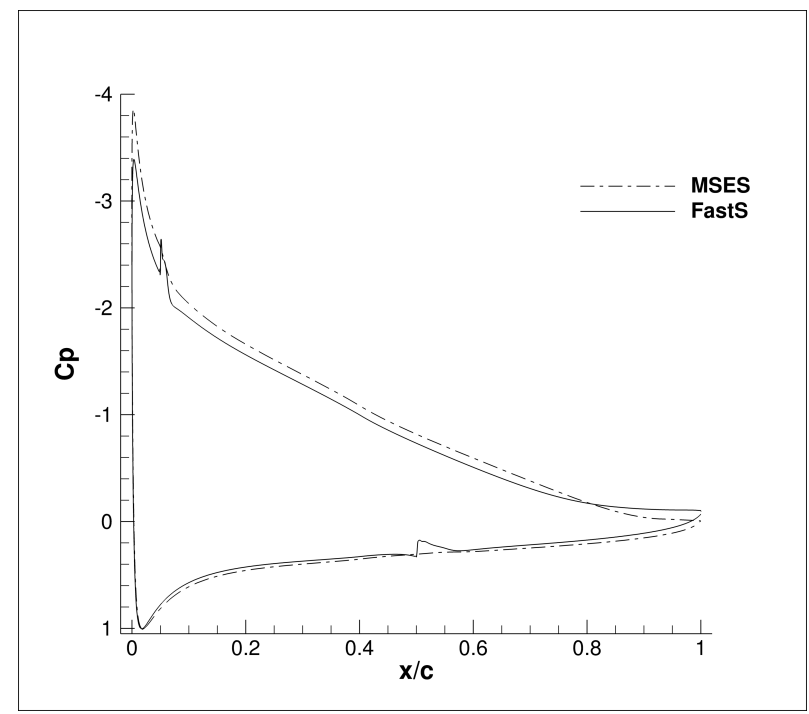

Figure 20: Comparison of mean Cp computed with FastS and MSES at $10 \mathrm{deg}$. incidence.

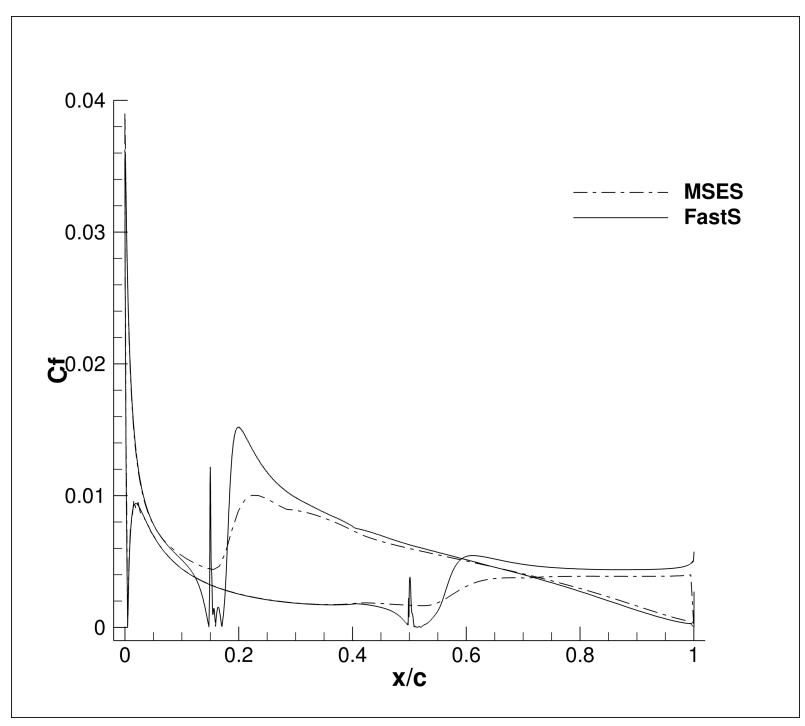

Figure 21: Comparison of mean Cf computed with FastS and MSES at $5 \mathrm{deg}$. incidence.

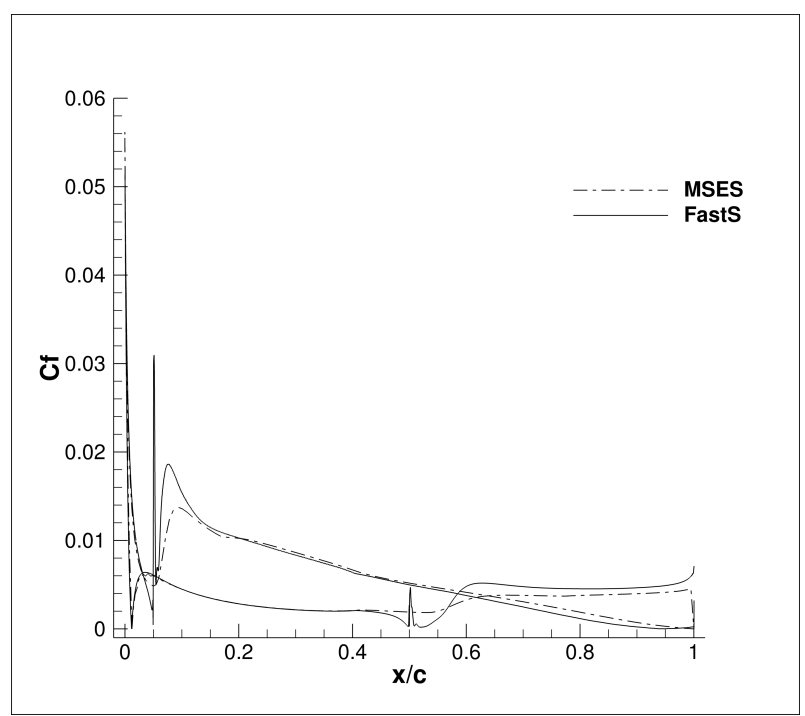

Figure 22: Comparison of mean Cf computed with FastS and MSES at $8 \mathrm{deg}$. incidence. 


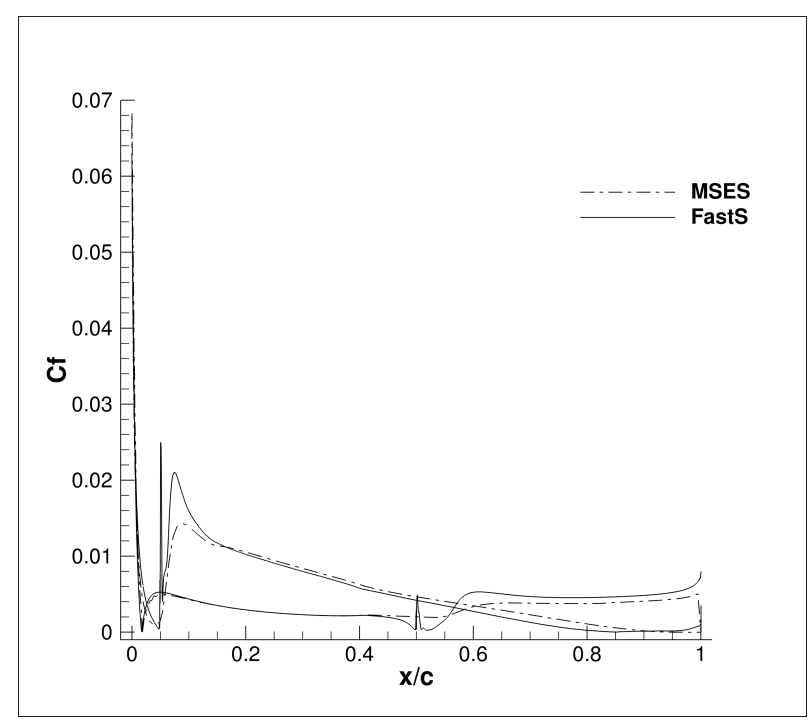

Figure 23: Comparison of mean Cf computed with FastS and MSES at $10 \mathrm{deg}$. incidence.

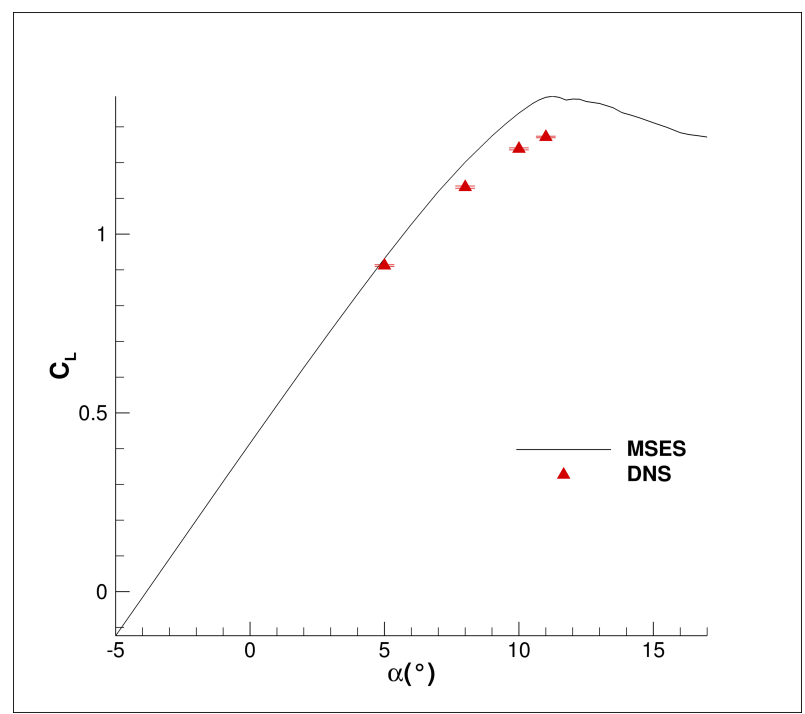

Figure 24: Comparison of lift polar computed with FastS and MSES.

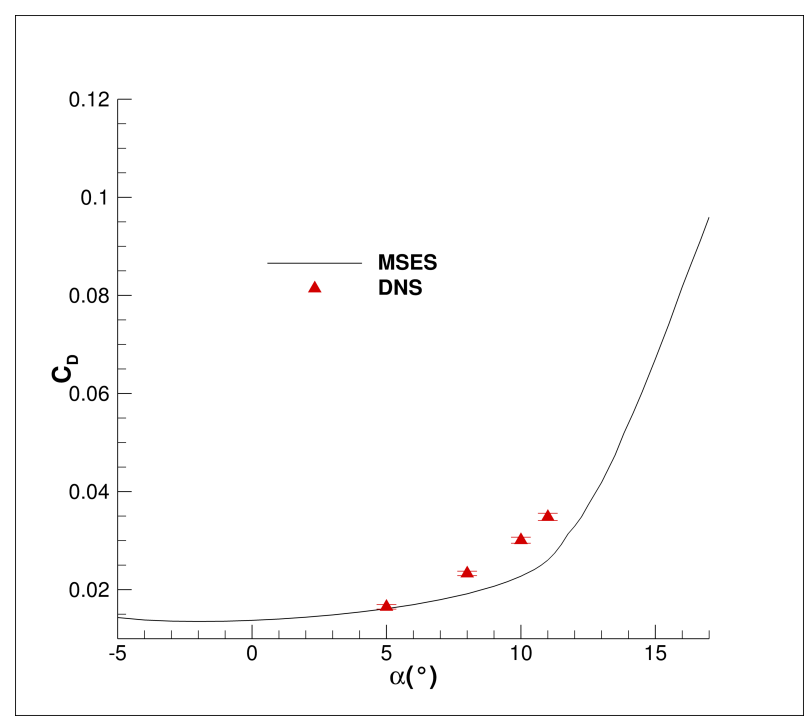

Figure 25: Comparison of drag polar computed with FastS and MSES. 